\title{
Sensor network architecture for a fully digital and scalable SPAD based PET system
}

\author{
Chockalingam Veerappan, Student Member IEEE, Claudio Bruschini, Member IEEE and Edoardo Charbon, Senior \\ Member IEEE
}

\begin{abstract}
Digital SiPMs in the recent past have emerged as a viable low cost alternative to PMTs providing higher granularity and MRI compatibility. The rich dataset generated by digital SiPM sensors have posed a challenge, especially at the system level when a multitude of such sensors are to be used. In this paper we present a sensor network based solution for data acquisition, scalable to multi-ring based pre-clinical, clinical and brain PET.
\end{abstract}

\section{INTRODUCTION}

Photonic sensors used in PET (Positron Emission Tomography) system have evolved over the years from PMT (Photomultiplier Tubes) to PSPMT (Position Sensitive Photomultiplier Tube) to Analog SiPM (Silicon Photo Multipliers), to address the need for increasing pixel granularity and timing response, while ensuring MRI compatibility and reduced size/cost. More recently, there has been growing interest in using CMOS integrated single-photon avalanche diodes (SPADs) based sensors [1] or digital SiPMs [2], [3].

The inherent digital property of SPADs and their migration to deep submicron CMOS processes have enabled the realization of digital photonic sensors with built-in intelligence [4]. Deep-submicron CMOS SPADs, along with 3-D integration will lead to highly granular pixel arrays, capable of time-stamping individual photons, the ideal sensing solution in PET systems. Digital SiPM based sensors, when used in PET systems, will generate hundreds of data for each potential gamma event, prompting the need to handle large data fluxes efficiently and accurately. Further, when using digital SiPMs, multiple rings of photonic modules are required to scale the dimensions of pre-clinical, clinical and brain PET systems. This work focuses mainly on techniques to handle the challenges associated with a data acquisition when hundreds of digital SiPMs are used in PET system.

Comprehending the challenges associated with the data acquisition and sensor control, a digital module known as Data Processing and Communication Unit (DPCU) facilitated with the data acquisition, processing and communication capability

This work is supported in part by the European Community within the FP7 SPADnet project.

Chockalingam Veerappan is with Delft University of Technology, Delft, The Netherlands (e-mail: c.veerappan@ tudelft.nl).

Claudio Bruschini is with EPFL, Lausanne, Switzerland (e-mail: claudio.bruschini@epfl.ch).

Edoardo Charbon is with Delft University of Technology, Delft, The Netherlands (e-mail: E.Charbon@tudelft.nl). was designed to reside at the back of every sensor tile. The photonic module thus realized has inbuilt intelligence of gamma detection, processing and communication.

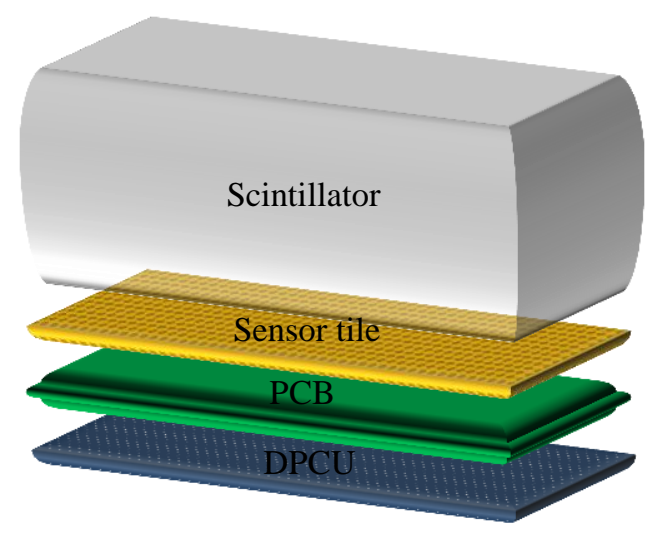

Fig. 1. Digital photonic module

To realize a PET system using digital photonic modules as described earlier, we propose a sensor network based design for data acquisition. In this approach photonic module comprising the sensor tile and DPCU forms a network node. This approach extends far beyond previously proposed data acquisition systems [5], [6] providing scalability and flexibility both in axial and in radial directions in terms of PET system construction.

In the proposed system, the data generated by each sensor tile is processed into data packets using DPCU. The generated data packets comprising the estimated values of energy, timing and spatial coordinates of the scintillation, are then used in communication with other nodes (other DPCUs) to perform coincidence detection and true event transfer in real time to an external (reconstruction) computer using the network.

\section{NETWORK ARCHITECTURE}

\section{A. Network Topology}

The photonic module acting as a network node is connected to its neighboring nodes forming a cylindrical mesh topology (Fig. 2) for a multi-ring based PET system. Further, to enable monitoring the status of the neighboring nodes, two communication links acting in opposite directions are used for inter-node communication. These communication links are specifically used for coincidence detection and true event transfer, respectively. In addition, a functional module called 
snooper, is used to collect and transfer data from the network to the external computer using a Gigabit Ethernet connectivity.

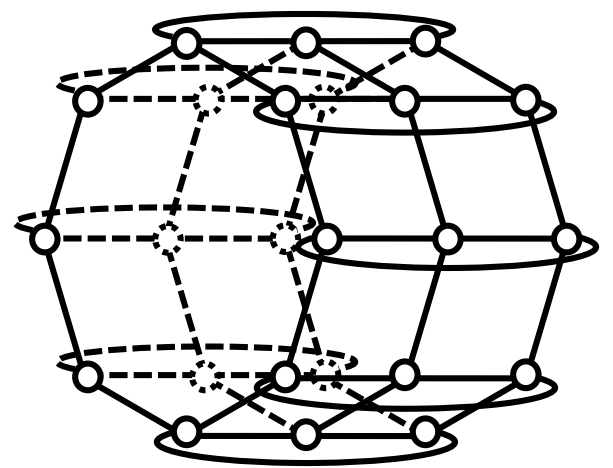

Fig. 2. Network topology for multi-ring PET systems.

The proposed network is designed to operate in two stages. In the first stage, coincidence detection is performed. In the second stage the detected coincidence events are paired to form a true event, and then transferred outside of the ring for further processing.

\section{B. Coincidence Detection}

To ensure network scalability, a distributed coincidence detection [6], [7] approach was chosen, whereby all network nodes are designed to perform coincidence detection simultaneously by comparing the locally generated events with the events that traverse the network nodes. In order to reduce the complexity in performing distributed coincidence detection, the network node was designed as shown in Fig. 3, to reduce the latency involved for a packet to identify its coincidence pair (if any).

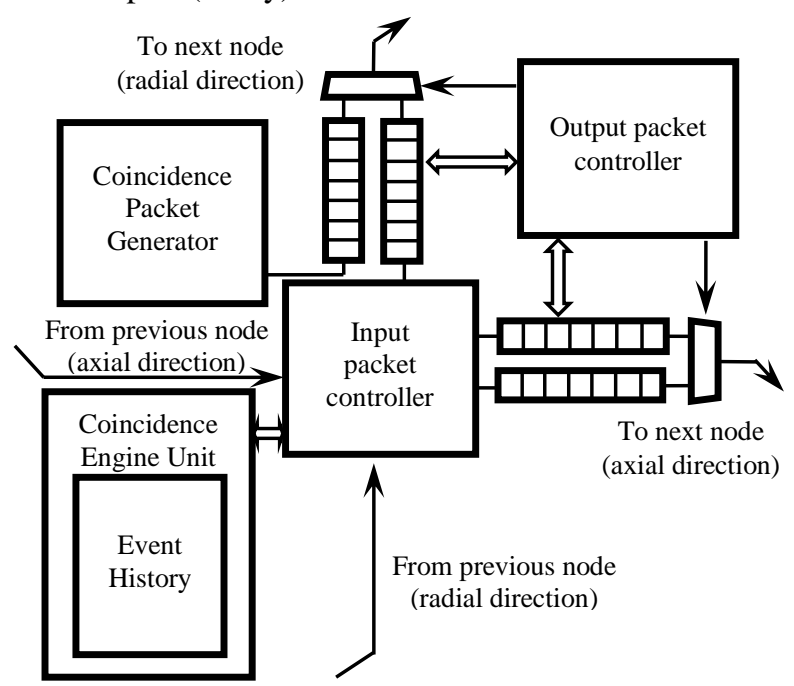

Fig. 3. Network node architecture for coincidence detection.

The use of smaller data packets or coincidence packets (CPs) of 32 bits (comprising the scintillation's timing and the node id), in combination with virtual channel (Fig. 3) based flow control techniques [8], [9] and an oldest-packet-firstscheduling algorithm, enhances the distributed coincidence detection performance. Furthermore, a static packet routing algorithm was used to reduce the packet latency. In this technique a CP traverses the network in clockwise direction until it reaches the end of the field-of-view. As the packet traverses the network nodes defined by the field-of-view requirements, a copy of the same is made to traverse the nodes present in the axial direction, thereby parallelizing the work load leading to an effective latency reduction.

\section{True event data transfer}

In case of a coincidence event, it will be present in two different nodes. To enable pairing these two events, the network is designed in such a way that the coincidence event with the node-ID larger than its pair is communicated to its pair using a static X-Y routing algorithm. The true event pair formed in this stage will then be transferred to the external computer via the snooper.

\section{Packet dropping probability}

For a network functioning as a data acquisition system, the packet dropping probability is a critical performance metric. In case of the PET application it is critical to ensure that all nodes maintain equal packet dropping probability all throughout the data acquisition duration. This condition will ascertain that at any point in time, no gamma event is lost while its pair is being processed in the network. To ensure equal packet dropping probability, a design strategy was devised for intra-node communication as well as for the internode communication. In this strategy, packets other than CPs are transferred to the next node only when the receiving node's network resource utilization is less than the current node. For intra-node communication a protocol was devised to allow transfer of data packets only when the receiving module is free to receive it. These two strategies work in conjunction to maintain almost equal packet dropping probability across all nodes.

\section{SiMULATION RESULTS}

A behavioral description of the network model developed in MATLAB was used to perform simulations.

\section{A. Coincidence Detection}

A set of simulations were carried out to understand the network's capability and to perform coincidence detection.

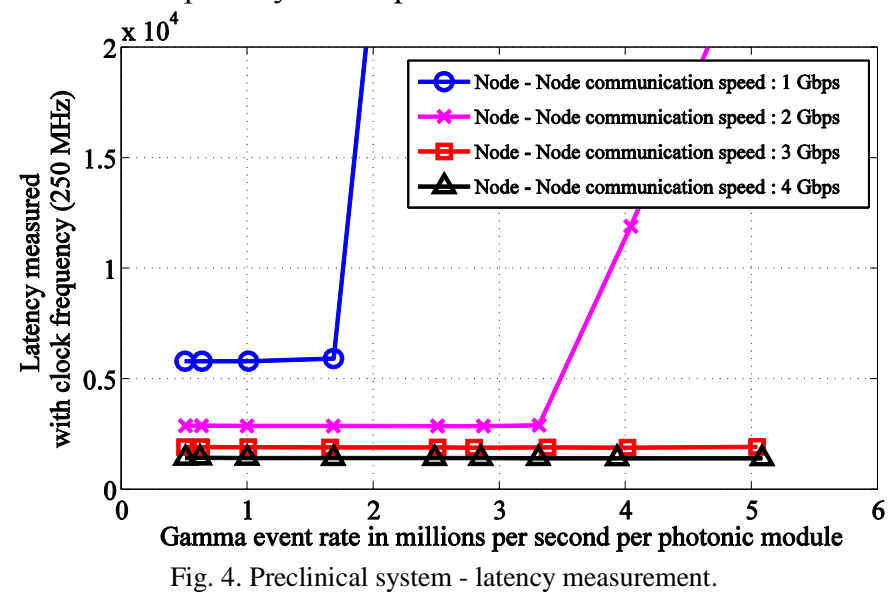




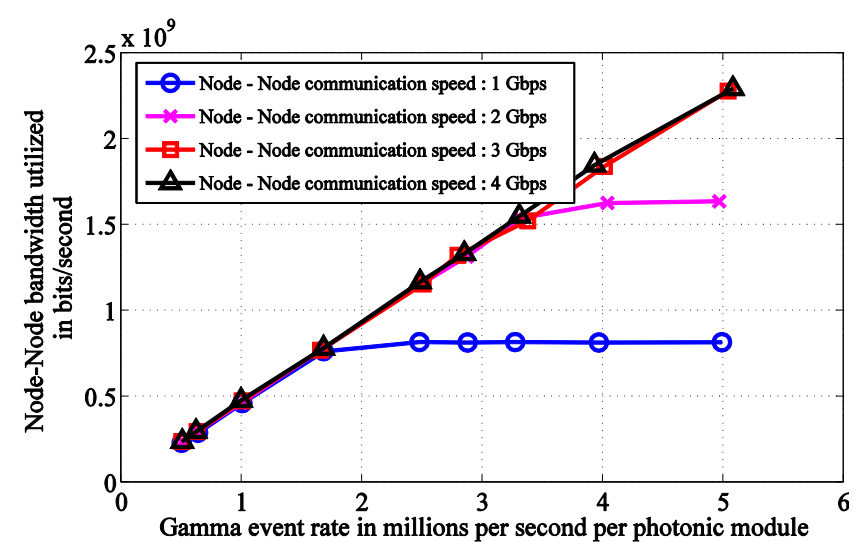

Fig. 5. Preclinical system - axial link bandwidth utilization.

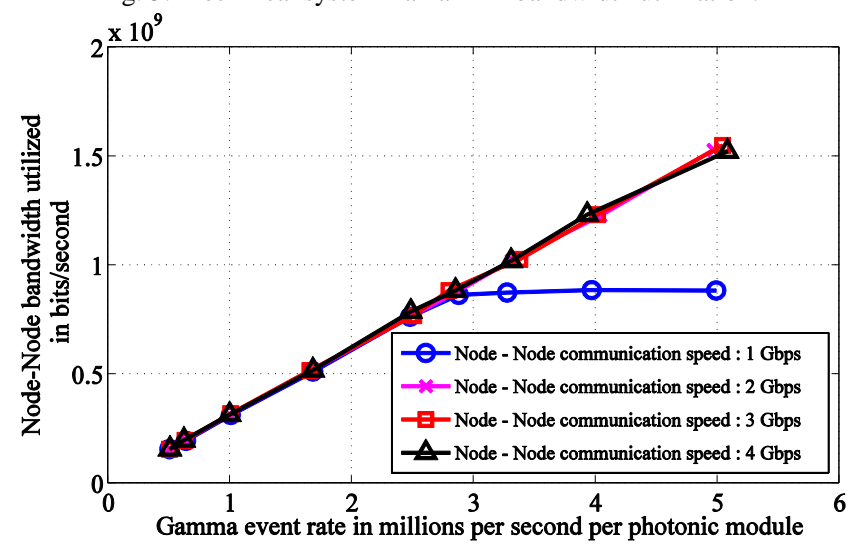

Fig. 6. Preclinical system - radial direction bandwidth utilization.

Simulation results in-case of pre-clinical dimensions (5 rings of 10 photonic modules each) is shown in Error! Reference source not found.-Fig. 6. In these simulations, latency involved for a coincidence packet to find its pair was monitored. For a network to be able to perform coincidence detection, convergence in packet latency is required. Simulation results have shown that in case of a preclinical system with a node-to-node communication speed of $1 \mathrm{Gbps}$ and $2 \mathrm{Gbps}$, packet latency was found to diverge when the incident gamma event per photonic module is around 1.6 million events per second and 3.2 million events per second respectively. The reason for the observed behavior is evident from the bandwidth saturation observed in communication link interlinking the nodes.

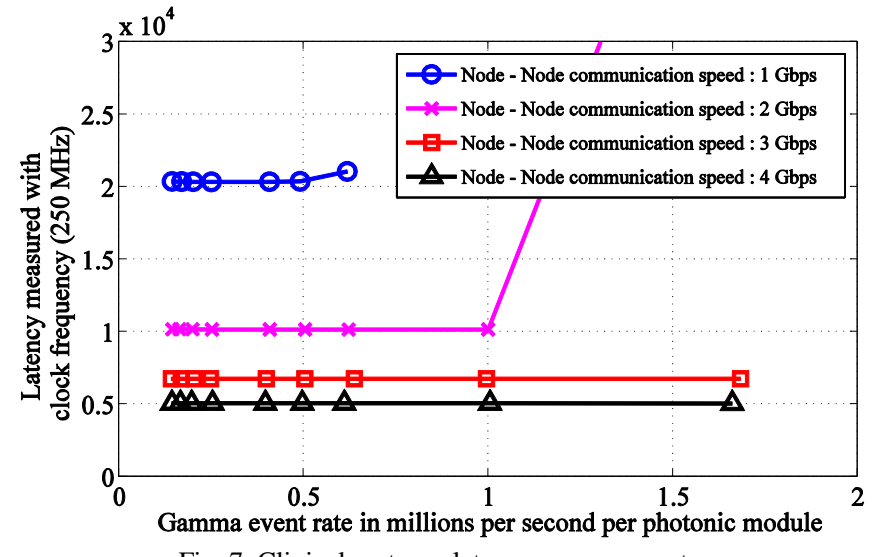

Fig. 7. Clinical system - latency measurement.

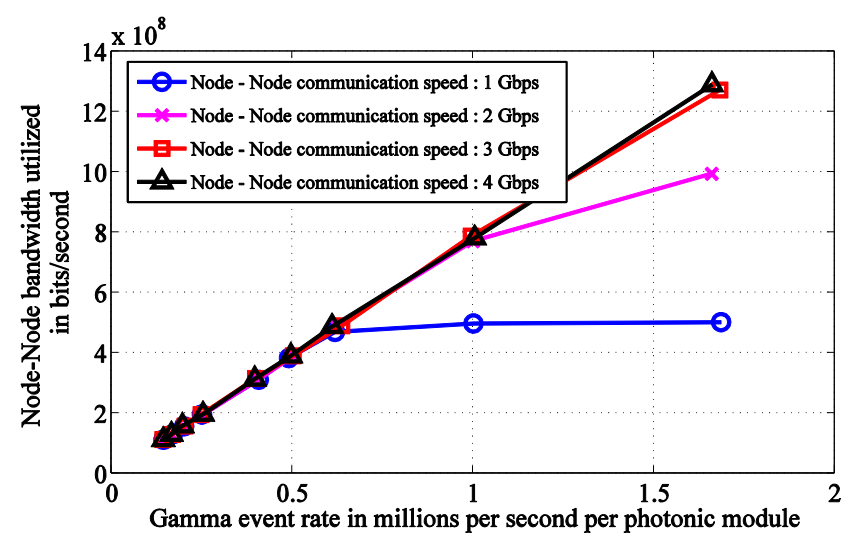

Fig. 8. Clinical system - axial link bandwidth utilization.

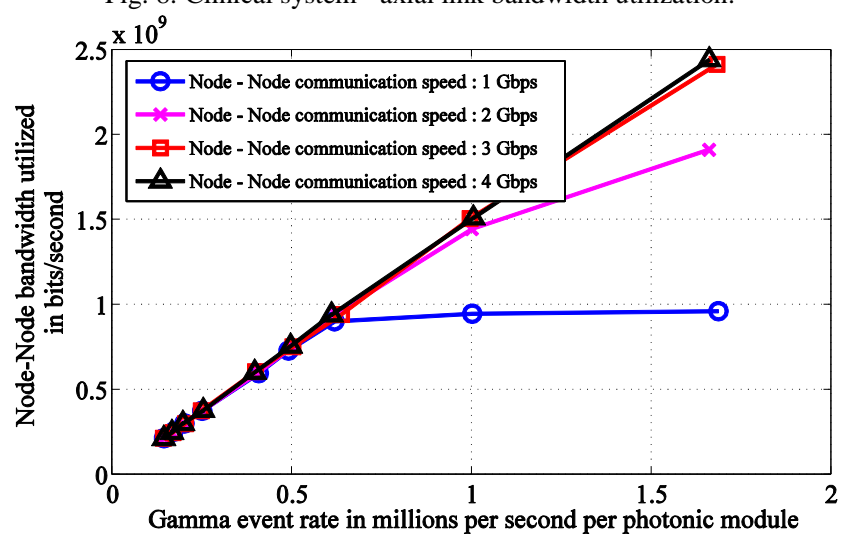

Fig. 9. Clinical system - radial direction bandwidth utilization.

For clinical (5 rings of 50 photonic modules each) and for brain PET (5 rings of 25 photonic modules each) dimensions, simulation results are presented in Fig. 7-Fig. 9 and Fig. 10Fig. 12 respectively. As observed in pre-clinical system simulations, in clinical and in brain PET, the packet latency was found to diverge when either the axial or the radial link bandwidth saturated. In-case of brain PET, the wider field-ofview requirements has led to bandwidth saturation earlier than in clinical system, although the brain PET is half the size of the clinical system.

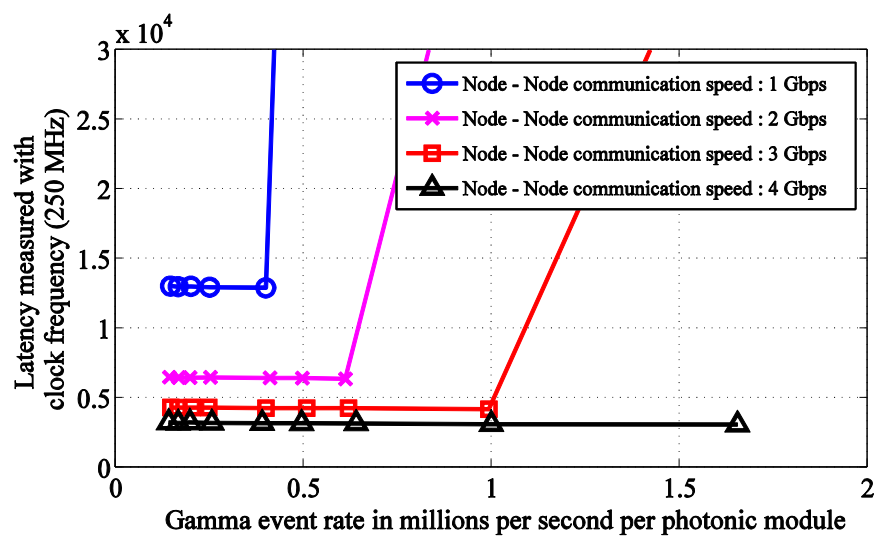

Fig. 10. Brain system - latency measurement. 


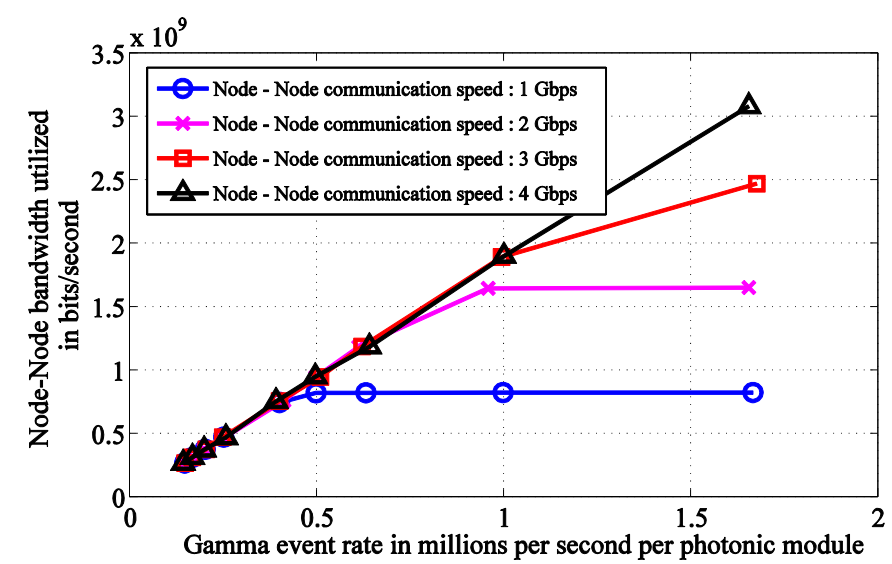

Fig. 11. Brain system - axial link bandwidth utilization.

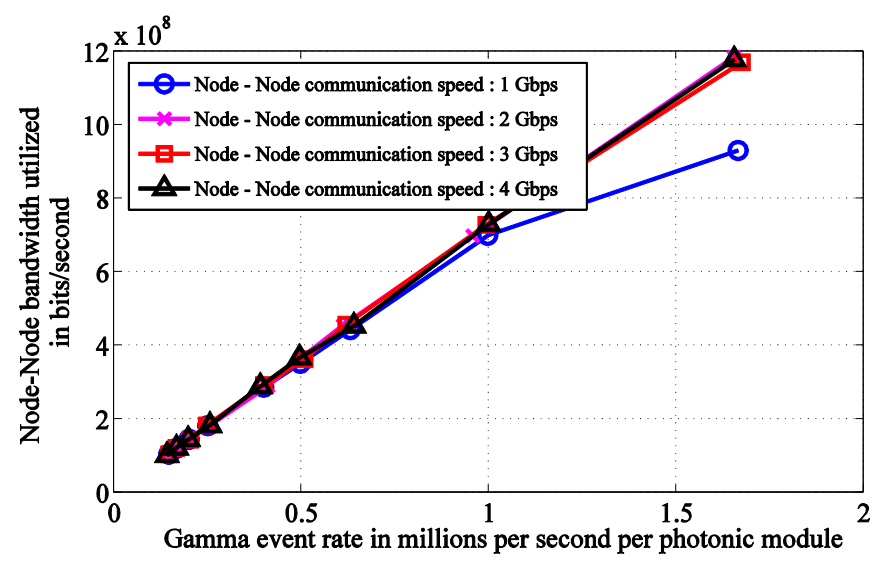

Fig. 12. Brain system - radial direction bandwidth utilization.

For digital photonic modules as envisioned in the SPADnet project [10], the maximum expected singles rates for preclinical, clinical and brain PET dimensions are of the order of $5 \mathrm{Mcps}, 500 \mathrm{kcps}$, and $500 \mathrm{kcps}$ respectively. These input data rates are far lower than the system's dynamic range, hence it can be ascertained that the presented network architecture will be capable of performing coincidence detection, provided the data communication rate reaches at least $3 \mathrm{Gbps}$. Achieving 3 Gbps data communication speed has been demonstrated to be feasible [11].

\section{B. Transient Simulations}

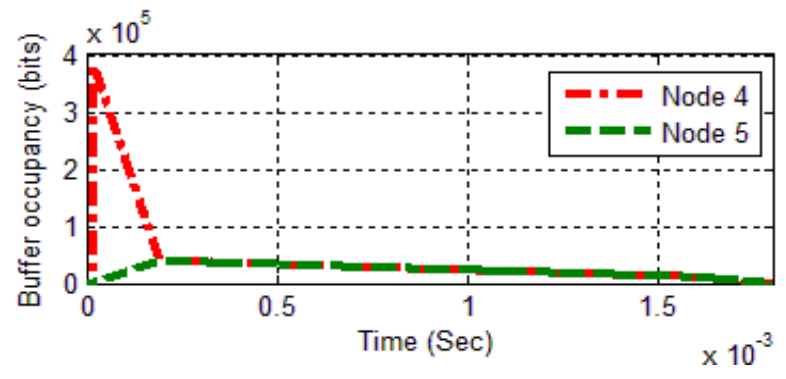

Fig. 13. Transient simulations.

Further, the data flow techniques proposed to maintain equal packet dropping probability across the system were tested by performing transient simulations. In this simulation, a huge number of packets were artificially injected into a given node (e.g. node-4) while the network was in a steady state, and then the network resource utilization was monitored. The simulation results (Fig. 13) have shown that the network is capable of readjusting itself when an imbalance occurs; however, the rate at which it readjusts itself depends on the data communication bandwidth.

\section{CONCLUSION}

The digital photonic modules designed using digital SiPM in conjunction with DPCU provides in-situ capability of data acquisition, processing and communication. Facilitating DPCU with networking and coincidence detection capability as elucidated in this paper, it is feasible to ease the realization of PET system by interlinking the digital photonic modules, following the network topology as explained in the paper, without the need for any other hardware modules.

The detailed study carried out at various abstraction levels using simulations has shown that the proposed approach could in principle be used without any modifications to multi-ring based pre-clinical, clinical and for brain PET studies.

\section{ACKNOWLEDGMENT}

Authors are grateful to Xilinx Inc. for FPGA donation and to Major Péter and Papp Zoltán from MEDISO for their valuable time in providing feedback and comments.

Disclaimer: This publication reflects only the authors views. The European Community is not liable for any use that may be made of the information contained herein

\section{REFERENCES}

[1] M.W. Fishburn et.al., "System Trade-Offs in Gamma-Ray Detection Utilizing SPAD Arrays and Scintillators," IEEE Transactions on Nuclear Science, vol. 57, no. 5, October 2010.

[2] L. H. C. Braga et al., "A CMOS Mini-SiPM Detector with in-Pixel Data Compression for PET Applications," in IEEE NSS-MIC , 2011.

[3] C. Degenhardt et al., "The Digital Silicon Photomultiplier - A novel sensor for the detection of scintillation light," in IEEE NSS-MIC, 2009.

[4] C.Veerappan et al., "A 160x128 Single-Photon Image Sensor with OnPixel 55ps 10b Time-to-Digital Converter," in ISSCC, 2011.

[5] D.F.Newport et al., "QuickSilver: A flexible, extensible, and high-speed architecture for multi-modality imaging," in IEEE NSS-MIC, 2006.

[6] E. Kim et al., "A New Data Path Design for a PET Data Acquisition System: A Packet Based Approach," in IEEE NSS-MIC, 2011.

[7] Blake E. Atkins et al., "A data acquisition, event processing and coincidence determination module for a distributed parallel processing architecture for PET and SPECT imaging," in IEEE NSS-MIC, 2006.

[8] William J Dally, "Virtual-Channel Flow Control," IEEE Transactions on Parallel and Distributed Systems, pp. 194-205, March 1992.

[9] Charles L Seitz et. al., "Deadlock free message routing in multiprocessor interconnection networks," IEEE Transactions on Computers, vol. C-36, pp. 547-553, May 1987.

[10] Fully networked, digital components for photon starved biomedical imaging systems. [Online]. http://www.spadnet.eu

[11] Xilinx. [Online]. http://www.xilinx.com/products/silicondevices/fpga/index.htm 